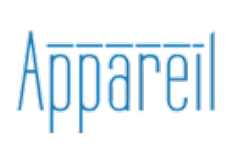

Appareil

$13 \mid 2014$

Trauma et création

\title{
Les métamorphoses de l'artiste après un coma
}

Réflexions d'un philosophe du vivant

\section{Georges Chapouthier}

\section{CpenEdition}

Journals

Édition électronique

URL : http://journals.openedition.org/appareil/2023

DOI : 10.4000/appareil.2023

ISSN : 2101-0714

Éditeur

MSH Paris Nord

Référence électronique

Georges Chapouthier, « Les métamorphoses de l'artiste après un coma », Appareil [En ligne], 13 | 2014, mis en ligne le 23 juillet 2014, consulté le 30 juillet 2020. URL : http://journals.openedition.org/ appareil/2023; DOI : https://doi.org/10.4000/appareil.2023

Ce document a été généré automatiquement le 30 juillet 2020.

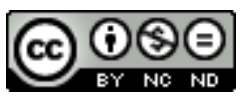

Appareil est mis à disposition selon les termes de la Licence Creative Commons Attribution - Pas d'Utilisation Commerciale - Pas de Modification 4.0 International. 


\title{
Les métamorphoses de l'artiste après un coma
}

\author{
Réflexions d'un philosophe du vivant
}

Georges Chapouthier

L'auteur remercie Lionel Naccache pour ses conseils.

1 Certains artistes découvrent de nouvelles voies ou une nouvelle sensibilité après un épisode de coma. Que peuvent en penser la biologie et la philosophie du vivant?

2 Les relations susceptibles de relier l'état du cerveau et celui de la pensée demeurent l'un des domaines fondamentaux les plus controversés de la philosophie. On sait notamment que se distinguent et s'opposent à ce propos des thèses matérialistes, qui voient dans l'activité cérébrale, elle-même conséquence des mouvements de la matière, l'origine stricte de toute pensée, des thèses idéalistes, qui, au contraire, voient dans les mouvements de la pensée l'origine première des mouvements de la matière et du cerveau, ou des thèses spiritualistes qui, sous diverses variantes, admettent une certaine indépendance entre activités cérébrales/matérielles et activités pensantes. Nous n'envisageons évidemment pas de résoudre ici cette question centrale et millénaire de la métaphysique !

3 Nous maintiendrons notre propos dans l'optique particulière de la science d'aujourd'hui. La neurobiologie moderne est, on le sait, d'inspiration matérialiste, c'està-dire qu'elle vise à interpréter les modifications de l'esprit strictement par les modifications sous-jacentes du cerveau, donc d'un système matériel, et c'est dans ce cadre philosophique et métaphysique, certes arbitrairement limité, que nous esquisserons notre discussion. Nous voudrions montrer qu'un tel positionnement philosophique, celui de la science d'aujourd'hui, est finalement la source de considérations fructueuses. Alors comment, dans cette optique, interpréter les changements brutaux de modes de pensée, les modifications, parfois spectaculaires, qui font qu'un artiste trouve, après une période de coma, voire un autre bouleversement psychique, l'accès à une nouvelle sensibilité ou à un nouveau mode d'expression? 


\section{Les métamorphoses du vivant}

4 Formuler un postulat d'interprétation matérialiste, c'est aussi se donner la possibilité de comparer aisément entre elles les structures perçues comme toutes d'origine matérielle, en l'occurrence de comparer aisément les modes de pensée à d'autres états de la matière, dont le cerveau est issu. Formuler ce postulat, c'est finalement vouloir enraciner les évolutions et les mouvements de la pensée dans l'évolution et le métabolisme des organismes vivants, dont le cerveau et la pensée sont issus. Rappelons, d'autre part, que des philosophes d'horizons métaphysiques variés, imaginent volontiers, sans pour autant partager tous les présupposés matérialistes de la science d'aujourd'hui, que la pensée puisse déjà se trouver, à l'état d'ébauche ou de germe, dans la plus petite quantité de matière, ce qui expliquerait, selon eux, qu'elle émerge ensuite, sous une forme développée, dans des organes complexes comme le cerveau. Un exemple en est fourni par Hans Jonas ${ }^{1}$. D'autres penseurs, comme feu le neurobiologiste Jean Delacour ${ }^{2}$, plaident pour un "matérialisme-identité », qui ferait de la matière et de la pensée deux aspects intrinsèquement liés d'une même réalité. Dans toutes ces optiques, considérer des ruptures brutales dans l'activité du cerveau, comme celles qui peuvent affecter un artiste après une période de coma, amène à renvoyer à d'autres ruptures brutales, beaucoup plus générales, que l'on rencontre dans la vie ou dans l'activité des êtres vivants qui peuplent la planète.

5 Or de telles ruptures sont nombreuses. Beaucoup d'organismes vivants subissent notamment ce qu'on appelle des métamorphoses, c'est-à-dire des bouleversements considérables dans leur forme, leurs fonctions physiologiques et leur mode de vie. L'une des métamorphoses les plus connues est celle qui transforme le têtard, une créature larvaire aquatique, dont le mode de vie est proche de celui des poissons, en une grenouille ou en un crapaud, qui sont des créatures adultes aériennes, au mode de vie proche de celui des reptiles. Encore plus frappante est la métamorphose bien connue de la chenille en papillon, qui nous servira ultérieurement d'exemple. La chenille, être rampant dans la dimension horizontale et focalisé sur la nutrition, donne naissance, après remaniement complet de ses organes, au sein d'un cocon protecteur appelé chrysalide, à un adulte aérien à vocation reproductrice et tourné vers la verticalité du vol, le papillon.

\section{Qu'est-ce que le coma?}

6 Mais, avant d'entamer ce retour hardi vers la biologie, précisons d'abord ce qu'est le coma, que nous avons pressenti comme le point de départ des nouvelles aptitudes de certains artistes. Chacun connaît, par expérience subjective, plusieurs états de conscience: la conscience attentive de la veille, la conscience diffuse de réveil, le sommeil et ses degrés de conscience variés, dont ceux qui révèlent une conscience gauchie, lors des épisodes de rêve, sans parler d'états de conscience plus particuliers, comme ceux qui accompagnent le somnambulisme, l'hypnose ou les hallucinations. Les degrés de conscience minimaux ne sont cependant pas ceux du sommeil, mais ceux d'un état pathologique qu'on appelle justement le coma.

7 Même si, étymologiquement, " coma " signifie en grec "sommeil profond », cet état pathologique doit être clairement distingué du sommeil normal, ainsi d'ailleurs que de la syncope (pathologique, elle aussi), qui est une perte de conscience brutale, en 
général de durée brève, et causée par des accidents vasculaires cérébraux. Le coma est un état plus ou moins prolongé, parfois fatal, avec perte de la conscience, perturbations graves des fonctions cérébrales, mais, sauf dans le stade le plus profond, maintien de la plupart des fonctions végétatives comme le rythme cardiaque ou la respiration. Le coma peut avoir des causes toxiques (abus de médicaments, d'alcool, de stupéfiants, absorption de pesticides, inhalation d'oxyde de carbone...), des causes métaboliques (diabète, insuffisance rénale, hyperthyroïdie...), des causes neurologiques (accidents vasculaires cérébraux, tumeurs, méningite, œdème cérébral aigu, épilepsie...) ou des causes traumatiques (traumatisme crânien). Par l'observation de l'activité électrique cérébrale (par un tracé qu'on appelle "électro-encéphalogramme » ou "EEG») et de diverses réponses corporelles, on distingue, au sein du coma, quatre stades de profondeur progressive. Lors du stade 4 , le plus profond, la vie ne peut se maintenir que par l'utilisation de moyens techniques et on parle parfois de « coma dépassé ».

8 En cas de retour à la vie consciente, les séquelles sont plus ou moins grandes selon la profondeur et la durée du coma. Elles sont d'ailleurs dues plutôt aux causes originelles du coma qu'au coma lui même qui, comme on l'a vu, est un état résultant de causes pathogènes. Les séquelles sont souvent négatives comme des difficultés sensorielles ou motrices générales, des troubles de l'intelligence ou de la mémoire. Les séquelles peuvent aussi être parfois (mais rarement) positives. Parmi ces dernières, on rencontre justement les cas des artistes qui, après une période de coma, découvrent une nouvelle sensibilité ou un nouveau mode d'expression. Que peut-on ajouter sur ces cas rares, mais spectaculaires?

\section{Les métamorphoses de l'esprit}

9 On doit d'abord relever que la science neurologique, dans son état actuel, reste à ce propos muette. Si l'on analyse la littérature scientifique, on ne trouve pratiquement aucun travail qui relate la manière dont on peut imaginer les processus cérébraux qui sous-tendraient les conséquences positives d'un coma. Si le neurobiologiste est alors désarmé et ne peut faire, comme d'ailleurs dans beaucoup de situations, qu'un simple constat sans véritable hypothèse explicative, le philosophe du vivant en revanche trouve, quant à lui, pour peu qu'il élargisse le débat, quelques leçons à tirer.

10 Le mouvement des idées mime le fonctionnement des organismes vivants. Plusieurs auteurs l'ont déjà remarqué, tel Jacques Monod quand il écrit : «Les idées ont conservé certaines propriétés des organismes. Comme eux, elles ont tendance à perpétuer leur structure et à la multiplier, comme eux elles peuvent fusionner, recombiner, ségréguer leur contenu, comme eux enfin elles évoluent et dans cette évolution la sélection, sans aucun doute, joue un grand rôle $»^{3}$.

11 Dans un essai de définition de l'architecture de la complexité, j'ai moi-même montré comment divers aspects de la complexité de la pensée ont une structure, dite «en mosaïque ", tout à fait analogue à la structure des êtres vivants. Dans les êtres vivants, comme dans d'importants secteurs de la pensée, la complexité se constitue en effet par "juxtaposition " d'éléments du même ordre de complexité, puis par "intégration » ultérieure de ces éléments, dans une structure plus complexe, qui, à l'image d'une mosaïque, laisse une certaine autonomie à ses parties. C'est le cas, parmi bien d'autres exemples possibles ${ }^{4}$, des cellules qui constituent un organisme ou des organismes qui constituent une population, mais aussi des éléments qui forment la mémoire ${ }^{5}$, la 
conscience $^{6}$, le langage... Ce dernier point, qui permet d'assimiler la juxtaposition puis l'intégration des mots dans une phrase à la construction d'un être vivant, a été développé en collaboration avec la linguiste Stéphane Robert. ${ }^{7}$

Revenons à nos artistes sortis du coma. Si nous poursuivons, à leur propos, une telle analogie, facilitée, comme on l'a vu, par une hypothèse de relation matérialiste entre le fonctionnement des organismes et celui de la pensée, force est de constater que les métamorphoses de la sensibilité artistique renvoient aisément, elles aussi, aux métamorphoses observées dans le monde vivant. Ainsi, si l'on souhaite encore développer l'analogie, le coma, état pathologique de repos corporel, de retrait plus ou moins prolongé et plus ou moins profond par rapport à l'activité normale du cerveau, jouerait le rôle protecteur joué par la chrysalide dans la métamorphose de la chenille. Les modifications éventuelles des connexions nerveuses après la période de coma, et leurs conséquences mentales vers de nouveaux intérêts artistiques, seraient les équivalents des bouleversements métaboliques qui surviennent lors des métamorphoses corporelles et tournent l'animal vers un nouveau comportement ou un nouveau mode de vie. Les métamorphoses de l'esprit, dont témoigne l'artiste après son coma, en développant un autre mode de création, seraient les transpositions, les analogues dans le monde de la pensée, des métamorphoses matérielles partout à l'œuvre dans le monde vivant et particulièrement dans le règne animal.

\section{Conclusion}

13 L'ancrage matérialiste, qui est celui de la biologie moderne, est, on l'a dit, nécessairement arbitraire sur le plan métaphysique et nous ne reviendrons pas sur ce point ; mais il est intéressant de constater qu'il permet ici une assimilation fructueuse et originale, qui compare le fonctionnement de la pensée au fonctionnement général de la matière vivante. Quelle que soit la nature même de la pensée, qu'elle soit, selon les philosophies ou les religions, une conséquence stricte ou non de l'activité cérébrale, force est de constater dans cet exemple que la pensée imite, dans ses mouvements, les mouvements $\mathrm{du}$ monde matériel. L'ancrage matérialiste offre donc une riche perspective évolutionnaire à la question des métamorphoses de l'esprit en les concevant comme des cousines des métamorphoses de la vie.

14 Ainsi les métamorphoses particulières de la pensée artistique, sur lesquelles nous avons été amenés à nous interroger, se présentent comme la continuation légitime des métamorphoses corporelles qui émaillent le règne animal depuis ses origines. L'ancrage matérialiste de notre propos donne à cette conception une réalité qui dépasse clairement le simple statut de la métaphore. Ainsi c'est d'une manière très concrète que l'artiste qui, après un coma, trouve une voie d'expression nouvelle, devient le lointain héritier de la chenille. C'est par le jeu de processus matériels similaires qu'il mime les remaniements complets du métabolisme des lépidoptères, pour s'envoler, dans le domaine esthétique... comme un papillon. 


\section{BIBLIOGRAPHIE}

Chapouthier Georges, L'homme, ce singe en mosaïque, Paris, Odile Jacob, 2001.

Chapouthier Georges, Biologie de la mémoire, Paris, Odile Jacob, 2006.

Delacour Jean, Une introduction aux neurosciences cognitives, Paris/Bruxelles, De Boeck, 1998.

Jonas Hans, Le phénomène de la vie (Vers une biologie philosophique), Paris/Bruxelles, De Boeck, 2001.

Monod Jacques, Le hasard et la nécessité, Paris, Seuil, 1970.

Robert Stéphane, Chapouthier Georges, «La mosaïque du langage », in Les origines du langage et des langues, Béatrice Fracchiolla (dir.), Paris, L'Harmattan, 2013.

\section{NOTES}

1. Hans Jonas, Le phénomène de la vie (Vers une biologie philosophique), Paris/Bruxelles, De Boeck, 2001.

2. Jean Delacour, Une introduction aux neurosciences cognitives, Paris-Bruxelles, De Boeck, 1998.

3. Jacques Monod, Le hasard et la nécessité, Paris, Seuil, 1970, p. 279.

4. Georges Chapouthier, L'homme, ce singe en mosaïque, Paris, Odile Jacob, 2001.

5. Georges Chapouthier, Biologie de la mémoire, Paris, Odile Jacob, 2006.

6. Georges Chapouthier, L'homme, ce singe en mosaïque, op. cit.

7. Stéphane Robert, Georges Chapouthier, «La mosaïque du langage », in Les origines du langage et des langues, Fracchiolla Béatrice (dir.), Paris, L’Harmattan, 2013, p. 197-209.

\section{RÉSUMÉS}

Si l'on se place dans le cadre des présupposés matérialistes de la science d'aujourd'hui, les bouleversements de la sensibilité artistique, qui peuvent apparaitre après un épisode de coma, prennent une signification biologique. Ils sont les échos, dans le domaine de la pensée, des métamorphoses corporelles que l'on rencontre chez les animaux dans le domaine de la nature.

\section{INDEX}

Personnes citées : Hans Jonas, Jean Delacour, Jacques Monod, Stéphane Robert

Mots-clés : matérialisme, biologie, métamorphose, coma, art 
AUTEUR

\section{GEORGES CHAPOUTHIER}

Neurobiologiste, épistémologue et philosophe, directeur de recherche émérite au CNRS. 